\title{
R\&D of PLC Teaching Simulation Training Platform Based on Unity 3D
}

\author{
Heyan Jiao, Huiyan $\mathrm{Li}^{+}$and Zhigang Bing \\ Tianjin University of Technology and Education, Tianjin Key Laboratory of Information Sensing \& \\ Intelligent Control, Tianjin, China
}

\begin{abstract}
Aiming at the problems of the long development time of the simulation visualization system and the shortage of PLC training equipment in colleges, this paper uses the virtual reality technology to develop the teaching simulation training platform by using the Unity 3D engine with the PLC for practical training. First of all, through the establishment of the model in 3ds Max and the development of Unity 3D, the virtual scene is completed. Then the communication between PLC and virtual platform is realized through serial communication. After testing several virtual scene topics, the platform is stable and reliable. At the same time, it can effectively simulate the operation of real equipment.
\end{abstract}

Keywords: Unity 3D, simulation training, virtual reality, serial communication

\section{Introduction}

For the implementation of the "Intelligent Manufacturing 2025" plan, promote the intelligent manufacturing, shorten the product development cycle, improve production efficiency and product quality, reduce operating costs and consumption of resources, speed up the development of intelligent manufacturing. It is of great significance to improving the adaptability and flexibility of the manufacturing supply structure and fostering new dynamics of economic growth.

At present, in the teaching of simulation training, the high-end products is less, the lack of interactive features, and the software of "demonstration" is more than "participation" software. Most products are single model and lack of hardware and software work together [1].

In the production practice in the field of automation, PLC (Programmable Controller) because of its simple operation, high reliability, strong function, has been widely used in intelligent manufacturing lines. In foreign countries, some top universities such as the University of Catania in Italy [2] and the University of Washington [3] have established open experimental teaching platforms. In the domestic, Tsinghua University has designed an experimental teaching platform based on automobile gearbox assembly production line [4], and Huazhong University of Science and Technology designed the intelligent transportation experiment teaching platform based on PLC [5]. However, comprehensive experiment platform based on PLC has many problems, such as take up a large field of experiment, multiple equipment sets, high platform costs, and safety issues in the teaching process. It is a lot of problems urgently to be solved in colleges in China.

\section{Design of Teaching Simulation Training Platform}

\subsection{Guiding Ideology of Platform Design}

The platform uses Unity 3D engine to build virtual objects instead of real objects. And by using dynamic environment model, 3D interaction design, virtual sensor and system integration technology, we completed

\footnotetext{
${ }^{+}$Corresponding author. Tel.: 13820966060

E-mail address:1hy2740@126.com.
} 
the construction of teaching simulation training platform. In the simulation learning environment, the teaching environment and teaching contents are simulated. Teaching and learning are no longer limited to tangible training places. The time and space of activities have been virtually expanded. Interactive mode of autonomous learning to improve the teaching and learning of the relationship between teachers and students, deepen students' understanding of theoretical knowledge, strengthen the practice ability training, and created a conditions for providing a teaching security, low cost, and cross space-time open simulation interactive learning environment.

\subsection{Design of Teaching Simulation Training Platform}

The teaching simulation training platform consists of the upper computer of the PLC simulation training software and the lower computer of the microcontroller main control chip. Overall design of the platform is shown in Figure 1. The PLC output module sends out the real physical electrical signal, which is uploaded to the upper computer by the serial communication module. The upper computer controls the real equipment in the virtual scene by parsing data packets, and sends packets through the virtual scene sensor. Through the communication module, the PLC inputs the real physical signals of the module to realize the operation of the simulation training platform. Infrared communication can also send data packets through remote control to control real devices in virtual scenes. In addition, there are industrial PROFIBUS, CAN bus and so on.

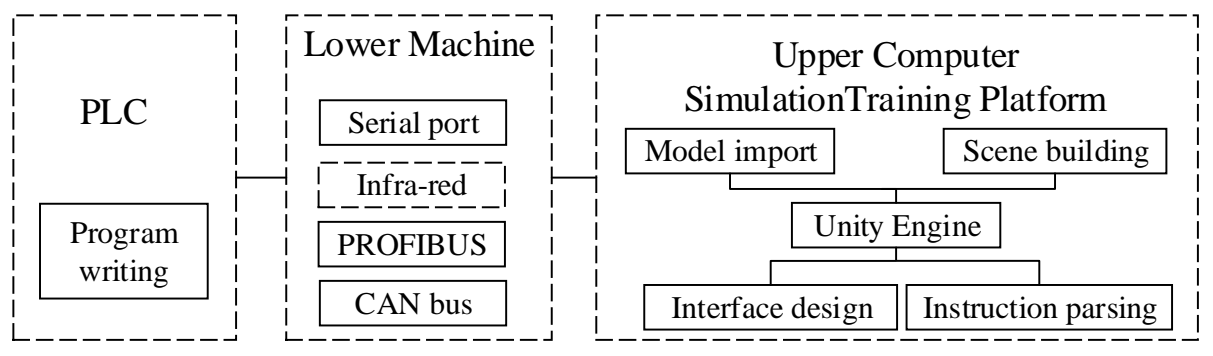

Fig. 1: Overall design diagram of platform.

\section{The Development of Teaching Practical Training Platform}

The development of teaching simulation training platform mainly includes four parts: 3D model building, user interface design, simulation drive, and hardware platform development.

\subsection{D Model Building}

At present, the 3D game engine mainly includes four game engines: Unity, UDK, UE4 and CryEngine. The Unity engine provides a very large game feature, and its scene is easy to use. Its best place is its cross platform, which means that the training platform can be quickly released to Android, IOS, Windows Phone8, and so on. And the Unity engine also supports a number of resource formats for 3D modeling software, such as 3ds Max, Maya, Softimage, Cinema 4D, etc., which makes it basically without model restrictions.

The virtual scene of the platform is drawn with 3ds Max. 3ds Max has a prominent advantage in the design of the model and conforms to the import requirements of the Unity engine. Therefore, the model is set up in 3ds Max and exported in .fbx format for use by the Unity engine. When modeling, we should try to make the models consistent with real equipment in shape and size. The authenticity and smoothness of interactive systems are important for user experience. The more detailed the model is, the more real it is. But the more data is generated, which affects the smoothness of the operation of the system, thus reducing the user experience. Therefore, it needs to optimize the model. In the case of no impact on the overall to minimize the model number and delete invisible surface and overlapping surface. In addition to minimize the use of Boolean command in order to reduce the number of avoid calculation error [6]. Finally export .fbx format file, import into Unity. 3D model construction directly affect the realistic degree of visual simulation. Through the design of special effects of the engine particle system, particle effects such as cloud, water, and flame are constructed. At the same time, add a sky box to construct the sky so that the edge of the terrain is closer to reality. 


\subsection{User Interface Design}

Good interface gives users good experience. Through the use of the built-in GUI and plug-in NGUI of Unity 3D [7], the interface design required by the simulation system is completed. Such as the description of the title in the question bank, and the attention and details of the operation. Each topic has a corresponding video demonstration, the students watch the operation video before use using it, which can be programmed more systematically.

\subsection{Simulation Drive}

As a simulation driver, the script of Unity $3 \mathrm{D}$ can not only realize the layout and rendering of the simulation interface, but also control the state and transformation of the object. In the simulation environment constructed above, the model is in a static state. The main function of the simulation driving is to allow the model to move dynamically and achieve the purpose of visualizing the simulation process. In the simulation training platform, considering the realization of serial data communication, the simulation driver uses C\# as a programming tool to complete the scripting of Unity 3D under Microsoft Visual Studio. The writing of a simulation driven script function includes scripting of the system entry interface, simulation settings interface and simulation interface. Take the driver script in the simulation interface as an example to introduce the main process of writing the Unity 3D driver script in .NET.

The Unity 3D serial port accepts the design pattern of multithreading and data, and avoids thread blocking with data structure queues. Set the serial number to COM5, the baud rate to 115200 , the data bit to 8, the check bit to IO.Ports.Parity.None, the stop bit to IO.Ports.Stop_Bts.One [8], and keep the same with the lower computer serial port. Open a thread to receive data through the Thread function. The data structure queue type FIFO (First Input First Output) stored the received data in the FIFO. The data packet format is shown in Table 1. The format of the data is sent in hexadecimal form.

Table 1: Data packet format

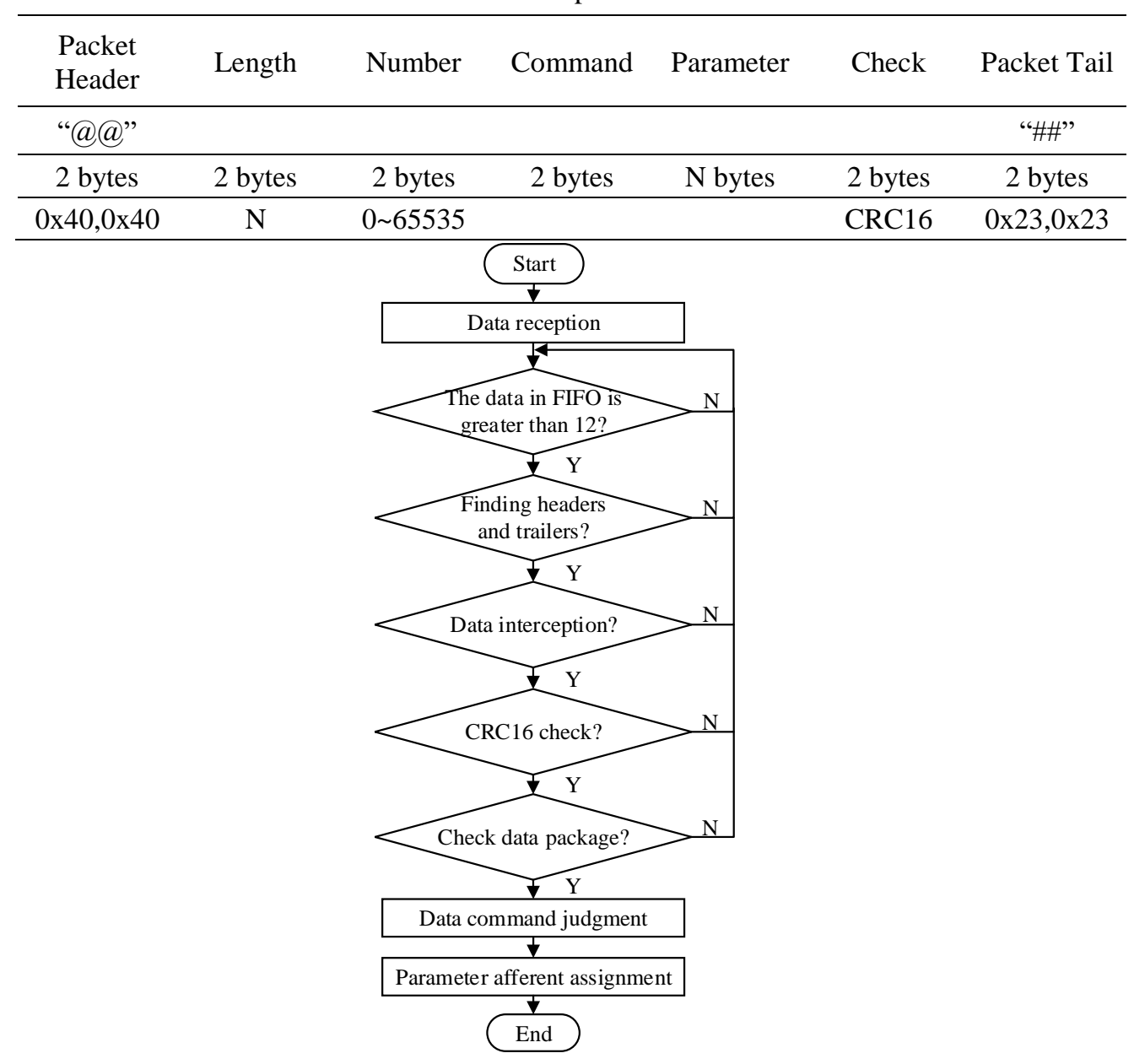

Fig. 2: Reading data Flowchart. 
In order to ensure the normal operation of the thread, the lower computer sends the heartbeat instructions at a rate of $10 \mathrm{~ms}$, and the upper computer receives the reply immediately. When the upper computer opens the serial port and thread, the data in the receiving FIFO is detected by the rate of $10 \mathrm{~ms}$. When the data length is greater than or equal to 12 bytes, the data is intercepted in FIFO. The intercepted data is judged by the header and tail as complete data, and then CRC16 checksum is used to determine whether the data is wrong data. According to the packet number in the data, real time judgment of the old and new. Parse the data command and parameters, and finally pass the parsed data into and assign values. The flowchart for reading data shown in Figure 2.

\subsection{Development of Hardware Platform}

The communication module selects STC32C5A60S2 as the main control chip. The FPGA chip is used to process input and output, AD, DA, PWM, and CNT data in parallel. The connection between MCU and PLC is electrical connection, communication module needs $5 \mathrm{~V}$ and $24 \mathrm{~V}$, PLC needs $220 \mathrm{~V}$ voltage, and the use of USB serial communication between the microcontroller and the PC to achieve signal transmission. The control signal of the PLC output is converted into a digital amount by a D/A chip. Then passed to the virtual scene of the PC machine to control the operation of the virtual device through the input and output port DIO. The virtual sensor signal will feedback PLC by the System.IO.Ports, and the closed-loop control of the System will be realized. The MCU detects the output signal of PLC and puts data in the data pool by cycle, then sends data to PC by serial port communication. The virtual reality system determines the received data and controls the virtual device to complete the related operation. The virtual sensor signal in Unity 3D is sent to PLC. After PLC receives the signal, it detects the operation of the virtual device and sends out the control signal to adjust accordingly, so as to realize the normal operation of the teaching training platform.

\section{Simulation Results}

The question bank of the teaching simulation training platform is shown in Figure 3. To test the feasibility of the test platform, use the three layer elevator logic control system and the temperature curve to describe the temperature box products as example. Firstly, we use virtual reality technology to build virtual scene, correctly connect the virtual scenes of PLC, communication module and PC. Then publish the virtual reality system built in Unity 3D software into '.exe' format, run PLC program, and finally record some effect diagrams.

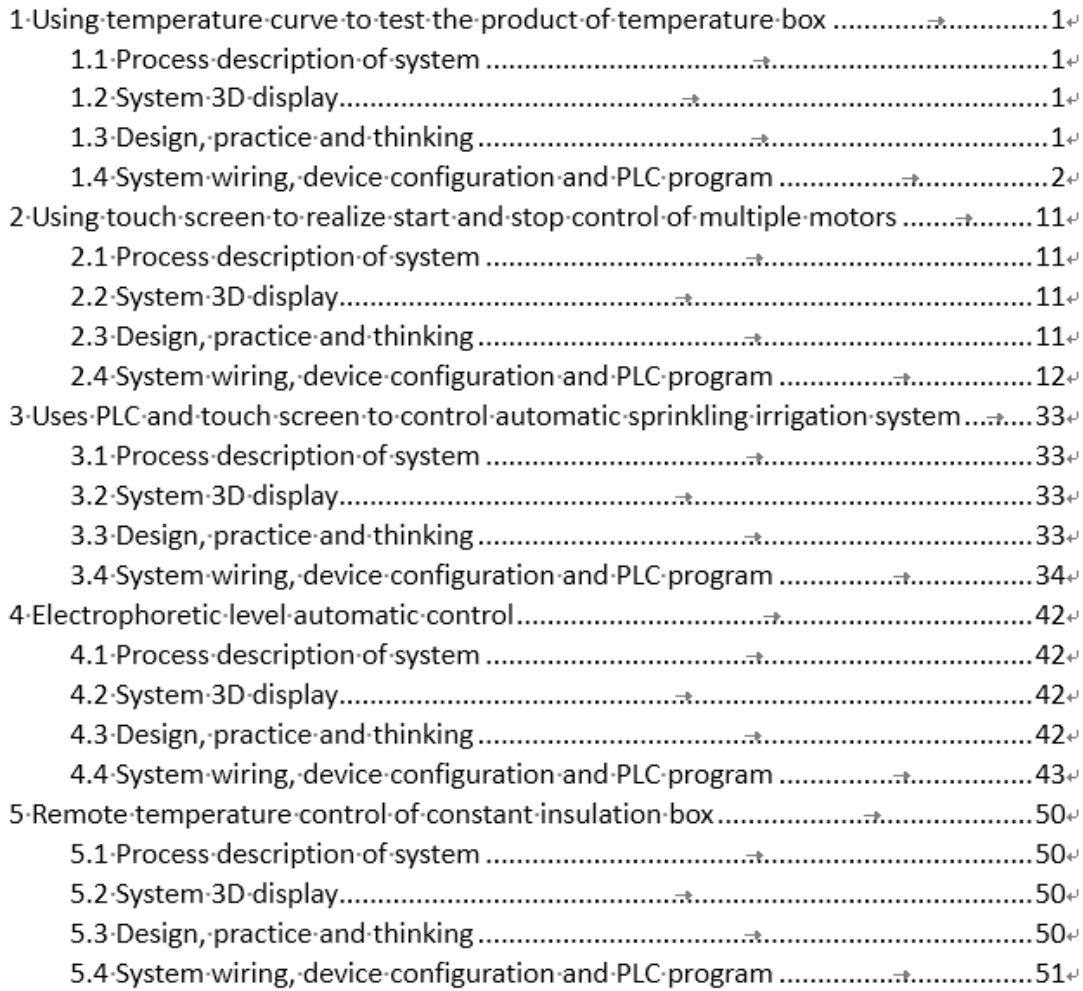

Fig. 3: The question bank of the teaching simulation training platform. 


\subsection{Three Layer Elevator Logic Control System}

Figure 4 is a three layer elevator logic control system.

(1) When the start button is clicked, the elevator can be located at any level. But if the flat layer signal is not detected, the elevator will go back to the bottom.

(2) The elevator responds to the signal when an external call signal arrives. Until the floor is reached, the elevator stops running, the doors open, and the door closes automatically after a period of time. The elevator responds to the call signal when an internal selection signal arrives. Until the floor is reached, the elevator stops running, the doors open, and the delay is automatically closed for a period of time.

(3) In the process of elevator operation, it will run to the top level. It can respond to two levels of upward external signal, but it doesn't respond to the two level down external signal. Until the elevator reaches the three level, it responds to the two level down external signal.

(4) When the elevator runs or does not reach the flat layer, the door button and the door button do not work. When the elevator is in a flat position and the elevator stops running, press the door button to open the door and close the door.

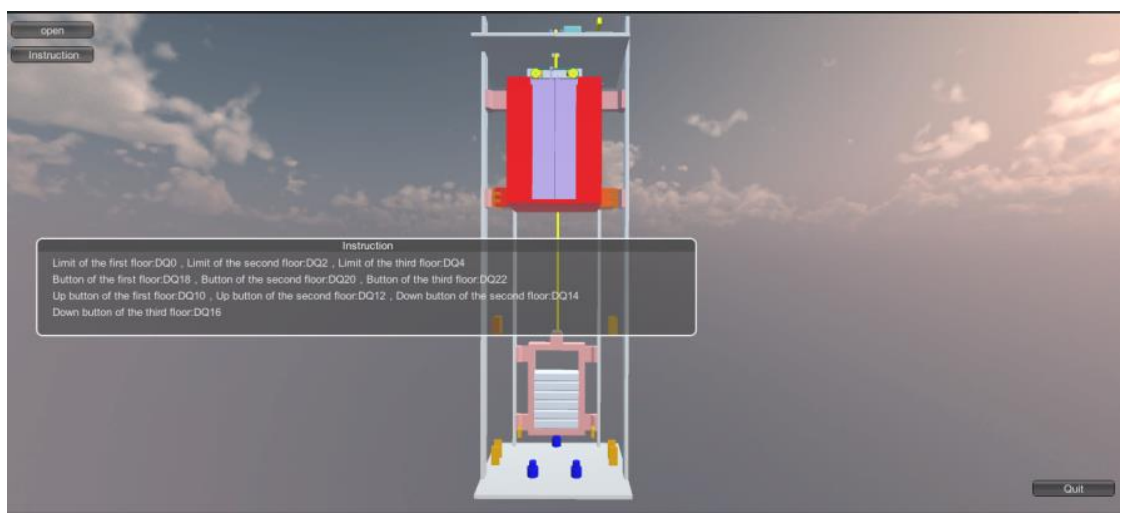

Fig. 4: A three layer elevator logic control system.

\subsection{Temperature Curve Describe of Temperature Box Products}

In order to describe the thermal insulation performance of the product, a detection device in the 3D scene is used to test, and the product grade is determined by calculating the temperature curve. As shown in Figure 5. Click the start button box in the heating rod heating when heated to a predetermined value. The system collects temperature every 5s. 10 times after the acquisition, it will generate a temperature curve. If the temperature is below the set value of the $85 \%$, that the insulation box is unqualified and buzzer alarm. If the temperature is higher than the set value $85 \%$, the product qualification.

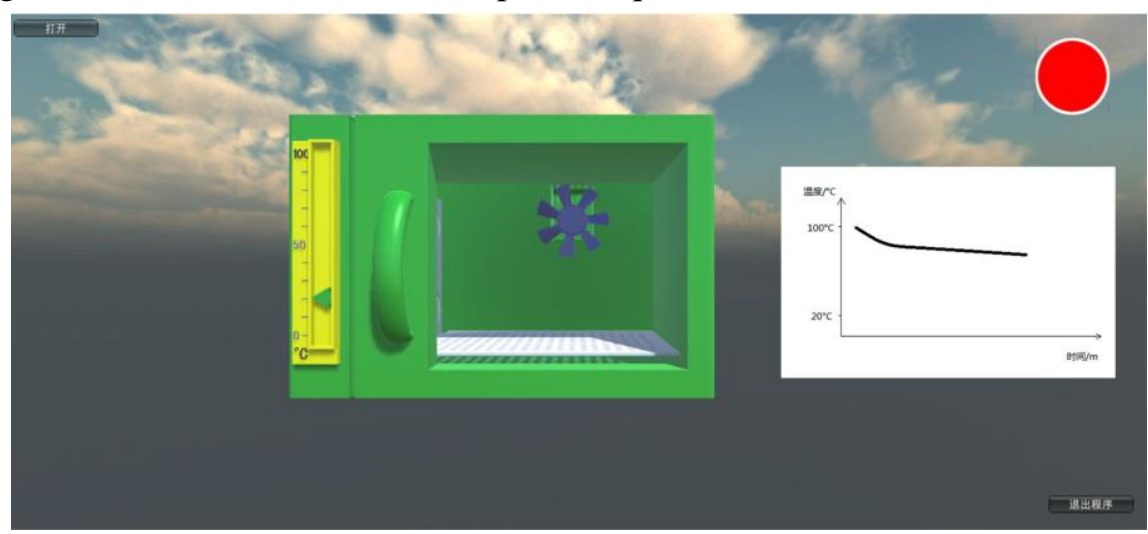

Fig. 5: Using temperature curve to describe the product of temperature box.

\section{Conclusion}

The test results show that the teaching simulation training platform runs smoothly, providing an intuitive, economic, safe and efficient environment for students to learn PLC, and solving the teaching difficulties of PLC training platform in some colleges. Through this platform, students can quickly and accurately write 
and debug the program, the dynamic analysis of various object performance, also can learn more about the various process work flow. Coupled with the real environment sound, can enhance on-site experience, stimulate interest in learning, improving learning efficiency. At the same time, it reduces the cost of training teaching in the same degree, avoids the damage of the equipment and the hidden danger of safety in the field, and has high economy and security. This platform will further improve, will update the server platform and docking database, rich content, increase of B/S platform and embedded PLC. It let the students not only can use in the laboratory, as long as the local network (such as classrooms, dormitories) can be used to facilitate learning, and make full use of resources; at the same time consider joining the 3D projection technology advanced 3D stereoscopic display, enhance the sense of immersion. So as to further improve the training platform and realize intelligence.

\section{Acknowledgment}

This work was supported by the National Natural Science Foundation of China (Project No. 61374182).

\section{References}

[1] Yanfen Li. Research on the construction technology of multi post and collaborative virtual production line. College of chemical engineering, Dalian University of Technology. 2009.

[2] Pitrone Nicola. Computer based tools for distribution network automation. 6th IASTED International Conference on European Power and Energy Systems. 2006: 161-166.

[3] Richter D. Infusing an interdisciplinary automation experience in engineering technology education. 114th Annual ASEE Conference and Exposition. 2007: 175-180.

[4] XuemeiHuang, Tianyuan Xiao. Research on the manufacturing system of intelligent reconfigurable automobile transmission assembly line. Mechanical science and technology. 2006, 25(4): 403-406.

[5] Qingxiu Feng, Junli Xia. Interactive PLC virtual experiment system based on OpenGL. Laboratory research and exploration. 2001, 30(2): 47-50.

[6] Xiaofei Zha. Research and development of Digital Mine 3D visualization system based on OpenSceneGraph. Henan Polytechnic University. 2001.

[7] Yusong Xuan. Unity3D game development. Post and post publication of the peopleagency. 2012.

[8] Dahu Wang, Haiyang Liu, Jingchong Wang. Development of the training system of Shearer Based on virtual reality. Computer Simulation. 2015, 32(6): 262-265, 283. 